\title{
Analysis for the structure of product manufacturing information flow of cloud manufacturing based on information measurement
}

\author{
Yi GAN*, Wei-ming HE**and Tohrub IHARA ** \\ ${ }^{*}$ College of Mechanical Engineering, University of Shanghai for Science and Technology, \\ 516 Jun Gong Road, Shanghai, 200093, P. R. China \\ E-mail: ganyi@usst.edu.cn \\ **Department of Precision Mechanics, Chuo University, \\ Kasuga, 1 Chome, Bunkyo, Tokyo, 112-0003, Japan
}

Received 25 November 2014

\begin{abstract}
In order to study the structural characteristics of product manufacturing information flowin cloud manufacturing from product angle, the mathematical description model of manufacturing information flow structure was defined. The existence of product manufacturing information flow conditions in the production process was described. Then the flow modes and special cases of product manufacturing information flow were analyzed. From an information-theoretic perspective, the Matter Information Quantity model was built including static entropy and dynamic entropy to describe the complexity of product manufacturing information flow quantitatively. To describe quantitatively the required information quantity in product manufacturing information flow states conversion, Distance of Information-state Transition model was established. Finally, an example is used to demonstrate the validity of the proposed methodology.
\end{abstract}

Key words : Cloud manufacturing, Product manufacturing information flow, Matter information quantity, Static entropy, Dynamic entropy, Distance of information-state transition

\section{Introduction}

In the 21 st century, the progress of technology and the diverse market demands make the product life cycle shortening, manufacturing industry is undergoing profound changes. Facing the limitless external diversification of products and the limitations of individual enterprise own production capacity, ASP(Application Service Provider), Manufacturing Grid, Agile manufacturing, Global Manufacturing and other network manufacturing modes has many limitations(Qi ,2003; Li, et al., 2010,2011; Zhang, et al., 2011.).

In order to reach the commanding height of advanced manufacturing industry, in 2011America government launched the plan of AMP (Advanced Manufacturing Partnership), and developed the development blueprint and the action plan of the intelligent manufacturing(SMLC. Implementing 21st century smart manufacturing report.2013). In 2013,Germany launched the Industrial 4 strategy by the expert group composed of the industry, government and research institutions(Securing the future of German manufacturing industry,2013), in order to improve the competitiveness of German industry and maintain the technology leadership, hoping reform the production process with internet of things and Internet service. Japan government was promoting the informationization of the manufacturing industry, information fusion physics system and large data applications. South Korea government launched the u-Korea plan to integrate the ubiquitous computing and the manufacturing system(Yao, et al.,2014). China research institutions and enterprises also actively explore the path of manufacturing upgrading(Xiong,2006; Zhou, 2013), and cloud manufacturing (CMfg) was proposed in 2009(Li,etal.,2010,2011). In the model of CMfg, manufacturing resources clouds formed with the massive resources of products total life cycle which are congregated by internet and being unified management and schedule. Manufacturing resources clouds can provide instant 
on-demand service.

CMfg caused extensive concern. To the service system, Tao et al. (2011) designed the function structure of the manufacturing cloud service management prototype system, and described the demand of cloud services combination for cloud manufacturing based on the whole life cycle of operation; Valilai et al. (2013) established the service oriented architecture, distributed collaborative manufacturing system platform based on STEP/XML standard, whose foundation is cloud computing technology. To the management and the use of CMfg resources, Luo et al.(2013) described a modeling and description method of multidimensional information for manufacturing capability in cloud manufacturing system; Laili Y. et al.(2012) proposed a model of optimal allocation of computing resources for cloud manufacturing systems. To CMfg application, the research object including mold parts database based on cloud services(Gu,et al,2012), intelligent grinding based on the cloud platform(Deng, et al.,2012), industrial cloud computing platform (Zeng, et al., 2012) etc.

According to above literatures, the research about CMfg focus on the prototype system, the top framework, the description and analysis of cloud manufacturing resource allocation, the parties of the cloud manufacturing and so on, lacking the study CMfg process from product perspective.

In the cloud manufacturing environment, the manufacturing of products processes has new features. (i) Subject to the rules of CMfg, the cloud manufacturing resource nodes can work independently which the manufacturing processes pass through. (ii) Under certain constraints, product manufacturing concurrent tasks can be achieved. And the situation that the product manufacturing concurrency processes competing on manufacturing resource nodes must be considered. (iii) Product manufacturing processes can run smoothly based on multiple cloud manufacturing resource nodes coordinated orderly. (iv) Product manufacturing processes continuous change dynamically in cloud manufacturing environment. And these changes must be communicated timely and effectively.

Product manufacturing processes of CMfg begin in converting the product demand information into product manufacturing information. With the services supplied by the CMfg and the interactions among material flow, energy flow, information flow and cost flow, the product manufacturing information will return to the market finally by the form of products meeting the demands. Follow the product manufacturing process, the logical product manufacturing information flows (PMIF) are formed transferring among the product manufacturing sectors.

Information is not energy or matter. It is a cognitive thinking mode based on material and energy. We can understand the world with the information displayed by physical movement (Zhang, 2003).PMIF is epistemological reflection and the abstraction to the product manufacturing flow, and it embodies the transfer processes and the attributes of product manufacturing information in the manufacturing processes. The transfer and processing of product manufacturing information constitute the basis of product manufacturing processes.

In this paper, the structure characteristics of PMIF in CMfg will be studied, and the state entropy and the information distance of the manufacturing information flow will be discussed.

\section{Mathematical description of PMIF structure}

PMIF in CMfg is not only the flow of product manufacturing traditional information between the manufacturing sectors; it emphasizes active, polymerization and dynamic. Ignoring the internal implementation of specific information processing functions, the complexity following the cloud manufacturing environment can be reduced. In this paper, the actual processing functions to the information were abstracted as functional nodes of product manufacturing processes, and the overall situation of manufacturing information processing and interaction is obtained.

\subsection{Algebraic description of PMIF}

To achieve the goal of product manufacturing, collaborative work among the product manufacturing processes is necessary. In CMfg, the manufacturing information flow of a product is denoted as MIF. The number of products manufacturing links with the ability of information processing of $M S$ is $M$, the set can be denoted as $M S=\left\{m s_{1}, m s_{2}\right.$, $\left.\ldots, m s_{M}\right\}$. The information flows sets depending on $M S$ are denoted as $I=\left\{m i_{1}, m i_{2}, \ldots m i_{N}\right\}$, where $N$ is the number of information flows. From the perspective of information processing, $M I F$ can be described by $M S$ and the corresponding $I$, which can be formulated as $M I F=<M S, I$.

Because of the diversity of information attributes, the individuals in different research fields have different 
cognition to the information. Based on the ontology (Yang, et al., 2010; Jiang, et al., 2010), the different links of information processing main domain have the same cognition to the same information in one research field. It means as follows.

$$
\forall m s_{k}, m s_{e} \in M S, \quad \forall m i_{n} \in I \quad, \quad m s_{k} \neq m s_{e} \rightarrow m i_{n}\left(m s_{k}\right)=m i_{n}\left(m s_{e}\right)
$$

In Eq. (1), $k \leq M, \quad e \leq M, \quad k \neq e, \quad n \leq N$.

In CMfg, the conditions for the PMIF existing are as follows.

$I(M I F) \neq \emptyset \rightarrow \exists m s_{k}, m s_{e} \in M S, \quad m s_{k} \neq m s_{e} \rightarrow|M S(M I F)| \geq 2$

Equation(2) means that the information difference among the manufacturing links is the driving force to product manufacturing information exchange.

If $M S(M I F)=1, M I F$ is not a PMIF and has been degenerated to a single product manufacturing links.

\subsection{Modes of PMIF}

PMIF of mechanical products are dynamic system composed by discrete events, and each node has input and output. To the node of $M S$ with the ability of information processing, the information flows input set of $M S$ is $I_{\text {in }}=\left\{I^{1}{ }_{\text {in }}, \ldots I_{\text {in }}^{r}\right\}$, where $r$ is the number of input flows, and its information flows output set is $I_{\text {out }}=\left\{I_{\text {out }}^{1}, \ldots I_{\text {out }}^{s}\right\}$, where $s$ is the number of output flows. The ability of information processing to $M S$ can be expressed as $I_{\text {out }}=M S\left(I_{\text {in }}\right)$, and can be illustrated by Fig.1(a). Going through different number of nodes, the complexity of the flow structure is not the same. In order to investigate the complexity of information flow, according to the relation of $I_{\text {in }}$ and $I_{\text {out }}$, there are 3 kinds flow basic modes and 2 kinds special cases of PMIF showing form Fig.1(b) to Fig1.(f).

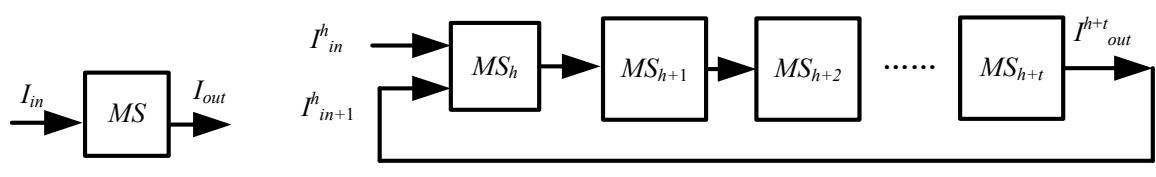

(a)

(b)

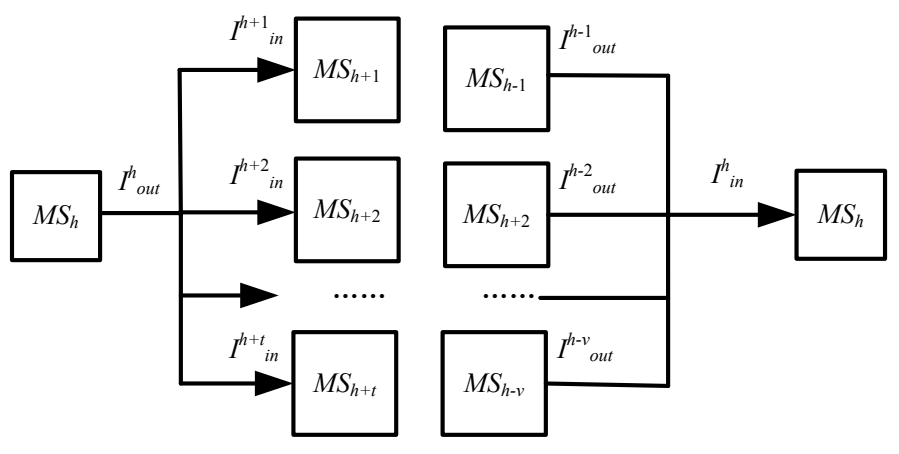

(c)

(d)

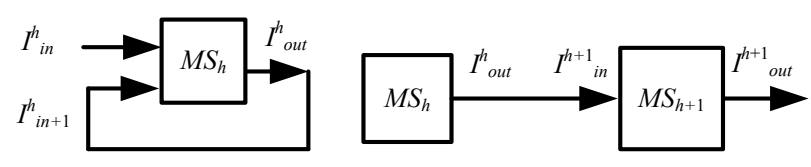

(e)

(f)

Fig. 1 .Illustration and the modes of the PMIF flow direction

Mode I. Information flow has feedback. The information flows through some links, closed information flow may 
be formed in these links. The only existing information flow passes into $M S_{h}$, through $M S_{h+1}, M S_{h+2}, \ldots$, and out from $M S_{h+t}$. The information flow being closed means $I^{h+t}{ }_{o u t}=I^{h}{ }_{i n+1}$, which shows as Fig.1(b).

Mode II. Information flow is distribution. The information flow is decomposed into plurality of input of subsequent links of $M S_{h+1}, M S_{h+2}, \ldots, M S_{h+t},(h+t) \leq N$. That is $I_{\text {out }}^{h}=I^{h+1}{ }_{i n} \cup I^{h+2}{ }_{i n} \cup \ldots \cup I^{h+t}$, , showing as Fig.1(c).

Mode III. Information flow is confluence. The output of pre order links of $M S_{h-v}, M S_{h-2}, \ldots, M S_{h-1}$ are confluence and pass through $M S_{h},(h-v)>0$. That is $I^{h}{ }_{\text {in }}=I^{h-1}{ }_{\text {out }} \cup I^{h-2}{ }_{\text {out }} \cup \ldots \cup I^{h-v}$ out , showing as Fig.1(d).

Special Case I. If information flow comes into being with only one link, there is the single link self feedbacks. It is the special case of Mode I. The information flows though $M S_{h}$, and the output is $M S_{h}\left(I^{h}\right.$ out $)$. The output information flows though $M S_{h}$ again and the input is $M S_{h}\left(I^{h}{ }_{i n+1}\right)$. That is, $I_{\text {out }}^{h}=I^{h}{ }_{i n+1}$, showing as Fig.1(e).

Special Case II. If information flow goes through only two links, mode II and mode III are degraded into special case of information flow with not change. After flowing though the link of $M S_{h}$, and the information flows though the next link of $M S_{h+1}$ without changes. That is, $M S\left(I^{h}{ }_{\text {out }}\right)=M S_{h+1}\left(I^{h+1}{ }_{\text {in }}\right), \quad I_{\text {out }}^{h}=I^{h+1}{ }_{\text {in }}$, showing as Fig.1(f).

In fact, the situations above do not appear alone, but occur at the same time, constituting the complex information flow.

\subsection{PMIF in CMfg}

Suppose there are $N u$ kinds of products manufacturing activities in CMfg, the products set is $P d=\left\{p d_{u}\right\}$, and the corresponding set of manufacture information set is MPI. Every kind of product develops orderly through the manufacturing links, forming their own manufacturing information flow.

There are three kinds of PMIF combination types according to the product manufacturing parallel tasks in CMfg (Li, et al., 2014). (i) Heterogeneous type. Each PMIF has own sub information flow sequence which are different to the others and do not share the candidate manufacturing cloud service sets. (ii) Homogeneous type. PMIFs have exactly the same sub information flow sequence and fully shared candidate manufacturing cloud service sets. (iii) Mixed type. PMIFs have a part of the same sequence and sharing some candidate manufacturing cloud service sets. Manufacturing tasks using manufacturing cloud services is long and monopoly (Zhang, et al., 2010; Jian, et al., 2010).So in this paper, each cloud service is shared by multiple PMIFs.

Each product having complex PMIF shown in Fig.1, different products information flow having the three kinds of combinations above, the complex PMIF network comes into being in CMfg. The PIMF network is denoted as Eq.(3).

$M P I=\left\{M I F_{u}\right\}, u=1,2, \ldots N u$

The changes of the manufacturing information in the PIMF network contain two different information quantities (Wang,2012). One is the information quantity that manufacturing information states have, which is called as Matter Information Quantity (MIQ). MIQ expresses the difference between a certain state and others. Another is the consumed information quantity when manufacturing information achieves the state, which is called as Distance of Information-state Transition (DIT). DIT distinguishes one from some of states. The following will discuss the two kinds of information quantities.

\section{MIQ model of PMIF}

\subsection{The complexity of PMIF}

CMfg is a complex system with competition and internal and external environment changing. The PMIF of CMfg also highlights the complexity of structure and operation. In 1948, Shannon (1948) first proposed that entropy is used to measure the information quantity. Deshmukh (1999) pointed out that the complexity of the manufacturing system is the states which are difficult to understood, described, predicted and controlled, and it is the expected information quantity to descript the states of the manufacturing system from the point of view of information entropy. Karp et al. (1992) thought that we can achieve the purpose of measuring and evaluating the states information of manufacturing resources, if the information quantity can be described which was required by manufacturing resources process structure and running states. Efstathiou (2002) proved that the system entropy is equal to the complexity of the system, and the system entropy can be used to describe and control the system states information quantity. Ko (2007) analyzed 
a design and development process of a product or a knowledge-base using complexity, which could be used as useful guidance in the product design and assembly planning.

According to the above, by measuring the information quantity contained in the PMIF links states, i.e., MIQ, the complexity of PMIF can be quantitatively discussed to predict and control the states of PMIF.

The complexity of PMIF is relative. PMIF with simple structure and small complexity is convenient for the whole system to be managed and controlled. But the simple structure is difficult to meet the changes of external demands. On the other hand, PMIF with large complexity can cushion the impact caused by the sudden changes of internal and external. At the same time, large complexity means uncertainty and poor predictability. PMIF with large complexity is hard to be managed and controlled.

Information is the opposite of entropy. Entropy is the measurement of the degree of confusion or the disorder degree of a system. But getting information can reduce the uncertainty, which reduces the entropy of the system.

Quantitative analysis the system states information by the measurement of PMIF entropy, it can help to more accurately understand the characteristics of complexity of the manufacturing system structure and the operation states.

The expected information quantity of MIQ to descript each PMIF link stationary state is called as static entropy, which is denoted as $E_{s}$. The actual needed information quantity of MIQ to descript each PMIF link evolution state is called as dynamic entropy, which is denoted as $E_{d}$. Just make sure $E_{s}$ and $E_{d}$ of each link, the operation condition of product manufacturing flow can be obtained.

\subsection{Static entropy model of PMIF}

A discrete random variable is $X=\left\{x_{1}, x_{2}, \ldots, x_{w}\right\}$, where $w$ is the number of elements of $X . x_{t} \in X, 1 \leq t \leq w$. The probability that $X$ takes the value of $x_{t}$ is $p_{t}$. Then the definition of $X^{\prime} \mathrm{s}$ information entropy is as Eq.(4).

$$
E(X)=-\sum_{t=1}^{w} p_{t} \mathrm{lb} p_{t}, p_{t} \geq 0, \quad \sum_{t=1}^{w} p_{t}=1, \quad \sum_{t=1}^{w} 1 \mathrm{~b} p_{t}=0
$$

In Eq. (4), $1 b$ means when $\log$ is given from the base of 2 to get the result with the same dimension with information entropy(Wang, 2006) whose unit is bit.

If $X$ represents a system, $x_{t}$ and $p_{t}(t=1,2, \ldots, w)$ respectively represent the probability of the system possible states and the probability of their occurrence, $E(X)$ is the information entropy of $X$.

$E(X)$ describes the degree of uncertainty to the system of $X$. The increase of the information quantity is based on information entropy reducing. More information obtaining, the information entropy is smaller, and the deterministic of system is greater.

According to the Eq. (4), $E(X)$ has the following characteristics.

(i) $E(X)=0$, when just only one of $p_{t}$ is 1 and all the other were 0 . This situation means that is complete certainty and information entropy of the system is minimization.

(ii) $E(X)=\lg w$, when all the states have equal probability, $p_{t}=1 / w$. This situation corresponds to the information entropy of the system is maximum. $X$ is maximum uncertainty.

(iii) $E(X)$ does not change if an additional state of $x_{w+1}$ is included in the system, with $p_{w+1}=0$.

Given a PMIF with $M \geq 1$ links, if the $m i$-th $(1 \leq m i \leq \mathrm{M})$ link has $S_{m i}$ possible independent states, based on the Eq.(4) the static entropy model for link $m i$ is,

$$
E_{s m i}=-\sum_{j=1}^{S_{m i}} p_{i j} 1 \mathrm{~b} p_{i j}
$$

In Eq.(5), $p_{i j}$ is the probability of state $j$ of link $m i .1 \leq j \leq S_{m i}, \quad \sum_{j=1}^{S_{m i}} p_{i j}=1$

Due to the properties of entropy, the static entropy model for PMIF is, 
$E_{s}=-\sum_{i=1}^{M} \sum_{j=1}^{S_{m i}} p_{i j} 1 \mathrm{~b} p_{i j}$

The result of Eq. (6) is the upper limit of PMIF static entropy. Considering the each manufacturing link states probability is not completely random, the actual information entropy is generally less than the upper limit value.

Calculating the static entropy of FMIF, the key is to determine the manufacturing links states. The identification of states depends on the research granularity on PMIF. And the research granularity depends on practical consideration such as system concerns and operability. To the discrete manufacturing system, information entropy of discrete events related to products evolution is calculated. For example, the events are the product converted from one node form to another or from a manufacturing state to another.

\subsection{Dynamic entropy model of PMIF}

The expected states of PMIF links are described by the static entropy. And the actual states are described by the dynamic entropy. According to direct observation, the actual states can be accessed. Based on Eq. (4) and Eq. (5), the dynamic entropy model of link $m i$ is,

$E_{d m i}=-\sum_{j=1}^{S_{m i}^{\prime}} p_{i j}{ }^{\prime} \operatorname{lb} p_{i j}{ }^{\prime}$

In Eq. (7), $S_{m i}^{\prime}$ is the actual states of link $m i, p_{i j}^{\prime}$ is the actual probability value of state $j$ of link $m i, 1 \leq j \leq S_{m i}^{\prime}$, $\sum_{j=1}^{S_{m i}^{\prime}} p_{i j}{ }^{\prime}=1$

There are two kinds of actual states of the PMIF links. One is controlled state according to plan, another is the out of control state such as manufacturing interruption and operating overtime. Let the controlled state probability is $p$, and the number of out of control states is $n i$, the dynamic entropy model for PMIF is,

$E_{d}^{\prime}=-p \mathrm{lb} p-\sum_{i=1}^{M} \sum_{j=1}^{n i} p_{i j}{ }^{\prime} \mathrm{lb} p_{i j}{ }^{\prime}$

In Eq. (8), the second item is the dynamic entropy due to the deviation from the expected state, which indicates the degree of uncertainty of PMIF links.

\section{DIT model of PMIF}

In product manufacturing, the information states change according to the manufacturing links changing. The information entropy can describe the information quantity of the states but cannot show the consumed information quantity to achieve the state. DIT can do it.

The information state transition is the changes among several possible information states of research object. DIT is the measurement of the obstacles which the research object meets during the process of its states transfer (Wang, 2012). The farther the information distance is, the more information needs to be acquired during the manufacturing process from one state of PMIF link to another (Gan, 2013A, 2013B).

Let $Y=\left\{y_{1}, y_{2}, \ldots, y_{\mathrm{n}}\right\}$ be the states set in PMIF, where $n$ is the number of states, $y_{t}$ is the $t$-th information state, $t=1$, $2, \ldots, n$. Let $p z_{t s}$ be the transition probability of the transfer which is from $y_{t}$ to $y_{s}(1 \leq s \leq n, s \neq t)$, and $D I\left(y_{t}, y_{s}\right)$ be the information distance of the transfer.$D I\left(y_{t}, y_{s}\right)$ is that $\log p z_{t s}$ to the base 2 , it has the same dimension with information entropy(Wang, 2006).

$$
D I\left(y_{t}, y_{s}\right)=\mathrm{lb}\left(1 / p z_{t s}\right)=-\mathrm{lb}\left(p z_{t s}\right), \quad t, s=1,2, \ldots, n, \quad \sum_{t, s=1}^{n} p z_{t s}=1
$$


For more information states transition, corresponding transition probability matrix $P$ and information distance matrix $D I$ are defined as follows:

$$
\begin{aligned}
& P=\left[p z_{i t s}\right]_{n \times n} \\
& D I=\left[\mathrm{lb} p z_{t s}\right]_{n \times n}
\end{aligned}
$$

For the series structure of the information transfer process in PMIF with $M$ links, showing as Fg.1 (b),(c), (d), the total transition probability is the product of every partial transition probability. For the parallel structure of the information transfer process in PMIF, showing as Fg.1(e),(f).

To the two states above, let $y_{00}$-th state be the total initial state, and $y_{n i}$-th state in the end be the final state. Let $p z\left(y_{i 1}, y_{i M}\right)$ be the total transition probability from the state $y_{i 1}$ to the state $y_{i M}$, and $D I\left(\left(y_{i 1}, y_{i M}\right)\right.$ be the total information distance. $p z\left(y_{i 1}, y_{i M}\right)$ and $D I\left(\left(y_{i 1}, y_{i M}\right)\right.$ are defined as follows:

$$
\begin{aligned}
& p z\left(y_{i 1}, y_{j M}\right)=\prod_{m=1}^{M} p z\left(y_{i m}, y_{j m}\right) \\
& D I\left(y_{i 1}, y_{j M}\right)=\sum_{m=1}^{M} D I\left(y_{i m}, y_{j m}\right)
\end{aligned}
$$

Generally, the number of cloud manufacturing service resources being enough, product manufacturing processes planning has multiple choices. The number of possible routes is $R$ which PMIF passing through and it will take on the multi- route structure of the information transfer process. Let $d_{r i}(r i=1,2, \ldots, R)$ be the information distance of the $r i$-th route, the total information distance is defined as follows:

$d=\sum_{r i=1}^{R} \omega_{r i} d_{r i}$

Where $\omega_{r i}$ is the weight of the $r i$-th route.

Let the initial of PMIF state be $y_{0,0}$, and the finial state be $y_{R, r i}$; the node $a_{r i}(r i=1,2, \ldots, R-1)$ passed through by PMIF has $b_{r i}$ states, and let $p w_{r i, r j}$ be the transition probability from the state $y_{r i, r j}\left(r j=1,2, \ldots b_{r i}\right)$ to the state $y_{r i+1, r j}$. The parameters of the information distance are represented in Table 1.

Table 1Parameters of the Information Distance.

\begin{tabular}{c|c}
\hline \hline Parameter Name & Parameter Value \\
\hline Path node & $a_{r i}(r i=1,2, \ldots, R-1)$ \\
\hline Number of states to each path & $b_{r i}(r i=1,2, \ldots, R-1)$ \\
\hline Information state of node $a_{r i}$ & $y_{r i, 1}, y_{r i, 2}, \ldots, y_{r i, b i}$ \\
\hline Transition probability from statey $y_{r, r}$ to state $y_{r i+1, r j}$ & $p w_{r i, 1}, p w_{r i, 2}, \ldots, p w_{r i, r j}$ \\
\hline States chain & $y_{1, r j}, y_{2, r j}, \ldots, y_{R, r j}$ \\
\hline
\end{tabular}

The information distance measurement for PMIF link states is defined as follows:

$$
\begin{aligned}
& \sum_{r j=1}^{b_{r i}} p w_{r i, r j}=1, \quad r i=1,2, \ldots, R-1 \\
& p z\left(y_{0,0}, y_{R, r j}\right)=p z\left(y_{0,0}, y_{1, r j}\right) \times \prod_{r i=1}^{R-1} p z\left(y_{r i, r j}, y_{(r i+1), r j}\right)
\end{aligned}
$$




$$
=p z\left(y_{0,0}, y_{1, r j}\right) \times \prod_{r i=1}^{R-1} p w_{r i, r j}
$$

$$
\begin{aligned}
D I\left(y_{0,0}, y_{R, r j}\right) & =-\operatorname{lb}\left[p z\left(y_{0,0}, y_{1, r j}\right) \times \prod_{r i=1}^{R-1} p w_{r i, r j}\right] \\
& =D I\left(y_{0,0}, y_{1, r j}\right)+\sum_{r i=1}^{R-1} D I\left(y_{r i, r j}, y_{(r i+1), r j}\right)
\end{aligned}
$$

Where $p z\left(y_{0,0}, y_{R, i}\right)$ is the total transition probability from the initial state $y_{0,0}$ to the final state $y_{R, r i}$, and $\operatorname{DI}\left(y_{0,0}, y_{1, r j}\right)$ is the total information distance.

In the condition of the multi-route information transfer, suppose there are $r m$ routes, and $\quad d r_{i d}(i d=1,2, \ldots, r m)$ is the distance of the $i d$-th route. Where $\omega_{i d}$ is the weight of the $i d$-th route, and $\sum_{i d=1}^{r m} \omega_{i d}=1$. The total information distance measurement $d r$ is defined as follows:

$$
d r=\sum_{i=1}^{m} \omega_{i} d r_{i}
$$

There are multi PMIFs in CMfg. Suppose $m$ products are corresponding to $m$ PMIFs, and $\quad d f_{i}(i=1,2, \ldots, \mathrm{m})$ is the information distance of every PMIF. Where $\lambda_{i}$ is the weight of the $i$-th PMIF, and $\sum_{i=1}^{m} \lambda_{i}=1$. The total information distance measurement is defined as follow:

$$
d f=\sum_{i=1}^{m} \lambda_{i} d f_{i}
$$

\section{An empirical study}

The information measurement of PMIF will be discussed in the part of this paper with an example as a refrigerator compressor motor development process. The main development processes of refrigerator compressor motor in a factory are follows. (i) The demands information includes the type, function and quantity of the motor. (ii) Design the prototype and get the preliminary design information. In the design process, designers exchange ideas forming the feedback of design link in PMIF. At the same time, the design department communicates with customers forming the feedback of design link and demand link in PMIF. (iii) Use electromagnetic analysis software to calculate and analyze the results of design, and get the analysis information. If the requirements of customers are met, it can be converted to processing information and the prototype can be trial production. If the requirements cannot be met, it should be redesign forming the feedback of analysis links and design link in PMIF. If customer requirements are unable to be met ultimately, the requirements may be changed or canceled, and the demand information is updated forming a feedback of analysis link and demand link in PMIF. (iv) With the customer confirmation, it can be mass-produced.

The PMIF of main development processes of refrigerator compressor motor in a factory is shown as in Fig. 2, which contains all kinds of the cases in Fig. 1

With the personalized and diversification of customer's demands, the gradually increased demands of products types and the shorten development cycle, the needs of market competition cannot be met by the factory original development process, the capacity of design resources and the manufacture resource.

Then the factory took part in a CMfg of refrigerator. By the CMfg, the more customers' demands can be acquired. Its design resources and manufacturing resources have become the CMfg service resources. It can use resources supplied by CMfg. Although the production process changed in CMfg to the factory, the frame of PMIF keeps the structure shown in Fig.2 from the perspective of the products, but the running environment is CMfg. 


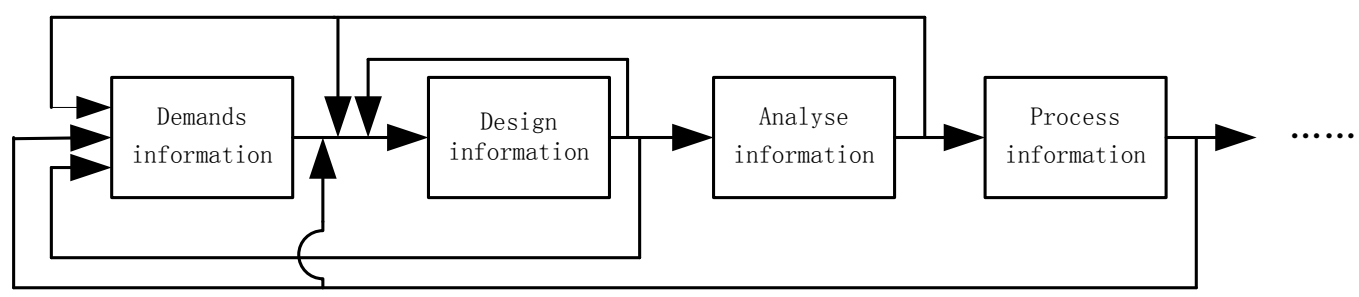

Fig. 2 The frame of the motor PMIF.

In order to calculate conveniently, we suppose the development processes obey the plan, so the static entropy and the dynamic entropy are equal and we only need to calculate one of them. And there are not feedbacks in PMIF.

(1) When factory did not take part in CMfg, there were two kinds of products demands information, denoted as ar1, ar2. The departments and information of design, analysis and processing have only one. The simplify PMIF is shown in Fig. 3(a).

Let the probability of $p_{i j}=0.5$ in demand link. And the state of other links is only 1 . According to Eq. (6) and Eq. (8), the state entropy of PMIF $E 1=1$ bit.

To the factory, all the demands must be met. Then the transition state of links is only 1. According to Eq.(17), DIT of PMIF DI1=0.

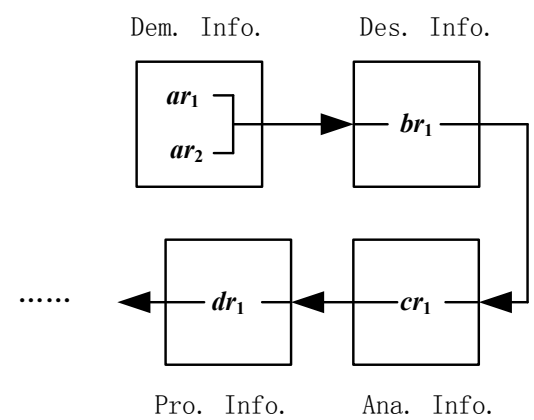

(a)

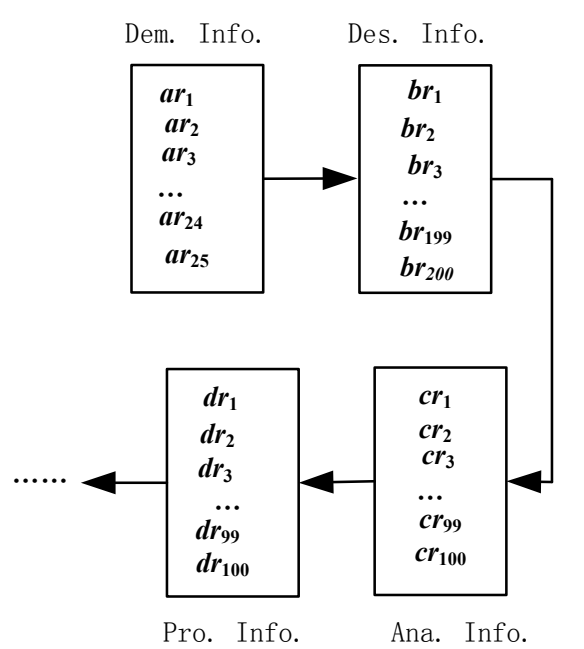

(b)

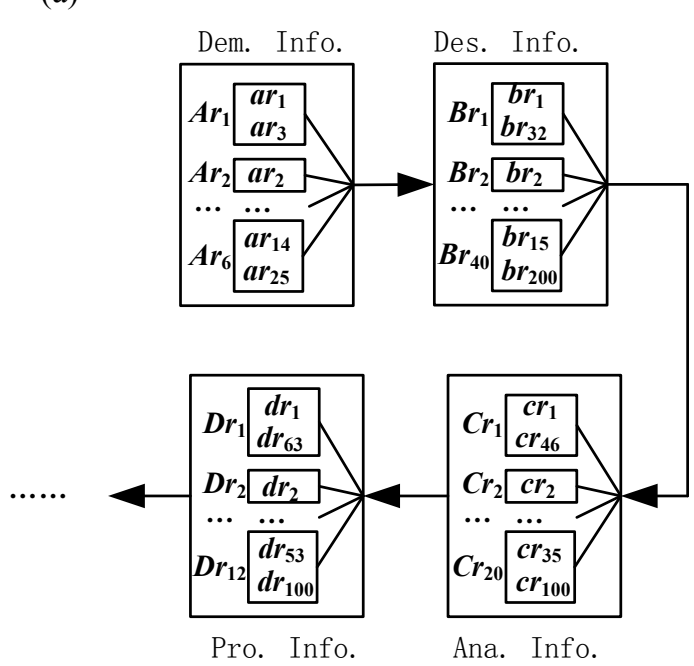

(c)

Fig. 3 The simplify PMIF.

(2) When the factory took part in CMfg, there were 25 kinds of products demands information of $a r_{1}, a r_{2}, \ldots a r_{25}$, 200 design departments being able to provide information of $b r_{1}, b r_{2}, \ldots b r_{200}, 100$ analysis departments being able to provide information of $c r_{1}, c r_{2}, \ldots c r_{100}$, and 100 pieces of process departments being able to provide information of $d r_{1}$, 
$d r_{2}, \ldots d r_{100}$. The simplify PMIF is shown in Fig. 3(b).

If CMfg is organized completely flexibly, the possible states (including work idle) of links are 26, 201, 101, 101, respectively. And the probability of one state of links are 1/26, 1/201, 1/101, 1/101, respectively. According to Eq. (6) and Eq. (8), the state entropy of PMIF can be calculated as follows:

$E 2=-\sum_{i=1}^{M} \sum_{j=1}^{S_{m i}} p_{i j} \mathrm{lb} p_{i j}=-\sum_{j=1}^{26} \frac{1}{26} \mathrm{lb} \frac{1}{26}-\sum_{j=1}^{201} \frac{1}{201} \mathrm{lb} \frac{1}{201}-\sum_{j=1}^{101} \frac{1}{101} \mathrm{lb} \frac{1}{101}-\sum_{j=1}^{101} \frac{1}{101} \mathrm{lb} \frac{1}{101} \approx 25.67 \mathrm{bit}$

In order to compare with before joining CMfg, PMIF only choose one resource status of each link. Then the transition probabilities in each link are equal. They are 1/25,1/200,1/100,1/100, respectively. According to Eq.(17), DIT of PMIF can be calculated as follows:

$D I 2=\mathrm{lb}(1 / 25)+\mathrm{lb}(1 / 200)+\mathrm{lb}(1 / 100)+\mathrm{lb}(1 / 100) \approx 18.93 \mathrm{bit}$

(3) Facing huge amounts of resources, CMfg optimize the management using the methods as task decomposition, resource classification, in order to play the benefit of resources sufficiently. If resources had been classified in CMfg, the products demands information became 6 classes $A r_{1}, A r_{2}, \ldots A r_{6}$, design departments information became 40 classes $B r_{1}, B r_{2}, \ldots B r_{40}$, analysis departments information became 20 classes $C r_{1}, C r_{2}, \ldots C r_{20}$, process departments information became 12 classes $D r_{1}, D r_{2}, \ldots D r_{12}$. The simplify PMIF is shown in Fig. 3(c).

With the classification of resources, the corresponding states are managed by category. The possible states (including work idle) of links are 7,41,21,13, respectively. And the probability of one state of links are 1/7, 1/41,1/21, 1/13, respectively. According to Eq. (6) and Eq. (8), the state entropy of PMIF can be calculated as follows:

$E 3=-\sum_{i=1}^{M} \sum_{j=1}^{S_{m i}} p_{i j} \mathrm{lb} p_{i j}=-\sum_{j=1}^{7} \frac{1}{7} \mathrm{lb} \frac{1}{7}-\sum_{j=1}^{41} \frac{1}{41} \mathrm{lb} \frac{1}{41}-\sum_{j=1}^{21} \frac{1}{21} \mathrm{lb} \frac{1}{21}-\sum_{j=1}^{13} \frac{1}{13} \mathrm{lb} \frac{1}{13} \approx 16.23 \mathrm{bit}$

The conditions is same as above (2), and the transition probabilities in each link are 1/7,1/40,1/20,1/12, respectively. According to Eq.(17), DIT of PMIF can be calculated as follows:

$D I 3=\mathrm{lb}(1 / 6)+\mathrm{lb}(1 / 40)+\mathrm{lb}(1 / 20)+\mathrm{lb}(1 / 12) \approx 15.81 \mathrm{bit}$

Based on the above results, we can conclude that PMIF in CMfg is much more complex than that in factory. And the complexity of PMIF in CMfg can be greatly decreased by effective resource management. With the example, the degree of decrease is:

$(E 2-E 3) / E 3 * 100 \%=58.16 \%$

By the same, the DIT of PMIF in CMfg is larger than that in factory. With the classification of resources, the degree of decrease is:

$(D I 2-D I 3) / D I 3 * 100 \%=19.73 \%$ 。

It is different from the decrease of the complexity of degree.

With the example, it shows that the complexity of PMIF and some state required of PMIF can be quantitatively described by the methods in the paper. This helps to master the operation of PMIF and provide data evidences to optimization for products development.

\section{Conclusion}

Following the product manufacturing process, the logical PMIF are formed transferring among the product 
manufacturing sectors. PMIF embodies the product manufacturing information transfer process and the manufacturing information activity characteristics. The conditions to the PMIF existing are there must be more than two information difference manufacturing links. In fact, the flow modes of distribution, confluence, feedback and so on, do not appear alone, but occur at the same time, constituting the complex information flow. MIQ of PMIF includes static entropy and dynamic entropy. The complexity of PMIF can be described quantitatively by making sure static entropy and dynamic entropy of each link. To describe quantitatively the required information quantity in PMIF states conversion, DIT should be calculated. DIT reflects the degree of difficulty of the states transformation. Though the MIQ and DIT of PMIF in CMfg is larger than that in enterprise, the enterprise can obtain more products demands and resources.

\section{Acknowledgment}

The research was supported by the National Natural Science Foundation of China (No.51375314). And thanks for the Chuo University for providing working conditions.

\section{References}

Deng Zh.H.,Liu W.,Wu X.X.,et al. Research and Application of Intelligent Grinding Cloud Platform Based on Cloud Computing. China Mechanical Engineering.Vol.23,No.1(2012),pp.65-68. (in Chinese)

Dretske F I. Knowledge and the Flow of Information. the David Hume Series, Philosophy and Cognitive Science Reissues.(1999) San Francisco: CSLI Publications.

Efstathiou J.The Utility of Complexity. Manufacturing Engineer,Vol.81,No.2(2002),pp.73-76.

Gan Y., Chen Sh.Sh., and Zhang X.M. Information State and Information Measurement for Modeling Design Based on TBS. China Mechanical Engineering.Vol.24,No.16(2013),pp.2131-2135. (in Chinese)

Gan Y.,Wang J.L.. Studies on Information States Measurement for Modeling Design. Applied Mathematics Information Sciences.Vol.7,No.2(2013),pp.627-632.

Gu X.J.,Huang Sh.Q.,Chen J.X.,et al. Cloud Manufacturing Service Platform Driven by Mold Manufacturing Industry Demand. Computer Integrated Manufacturing Systems.Vol.18,No.7(2012),pp.1650-1656.

Jian, H.,Bastani, F. B.,Yen, I. L., et al.. A Framework for Efficient Service Composition in Cyber-Physical Systems. In Service Oriented System Engineering (SOSE), 2010 Fifth IEEE International Symposium on, IEEE,(2010),pp.291-298.

JiangY., Peng G., Liu W. Research on ontology-based integration of product knowledge for collaborative manufacturing. The International Journal of Advanced Manufacturing Technology, Vol.49,No.9-12(2010), pp.1209-1221.

Karp A, Ronen B. Improving Shop Floor Control: an Entropy Model Approach. International Journal of Production Research,Vol.30,No.4(1992),pp.923-938.

Ko, Kwang Hee, Kishore Pochiraju, and Souran Manoochehri. Dynamic Evolution of Information Complexity for Analysis of Design and Development. Journal of Advanced Mechanical Design, Systems, and Manufacturing. Vol.1,No.1 (2007),pp.36-47.

Laili Y., Tao F., Zhang L., et al. A study of optimal allocation of computing resources in cloud manufacturing systems. The International Journal of Advanced Manufacturing Technology.Vol63,No.5-8(2012), pp.671-690.

LiB.H.,Zhang Li.,REN L.,et al. Further discussion on cloud manufacturing. Computer Integrated Manufacturing Systems.Vol.17,No.3(2011),pp.449-457.

LiB.H.,Zhang L.,WANG SH.L.,et al. Cloud manufacturing: a new service-oriented networked manufacturing model. Computer Integrated Manufacturing Systems.Vol.16,No.1(2010),pp.1-7,+16.(in Chinese)

Li X.B.,Yin Ch.,and Yin Sh.. Semantic description and characteristics of machine tool resources in cloud manufacturing environment. Computer Integrated Manufacturing Systems. Vol.20,No.9(2014),pp.2164-2171.

Luo Y.L.,Zhang L,Tao F,et al.A modeling and description method of multidimensional information for manufacturing capability in cloud manufacturing system. Int .J. Adv. Manuf. Technol.Vol.69,No.5-8(2013),pp.961-975.

Qi C.Q., Introduction to Manufacturing Industry Informatization (2003). China Astronautic Publishing House.(in Chinese)

Securing the future of German manufacturing industry: Recommendations for Implementing the Strategic Initiative 
Industrie 4.0. <www.acatech.de>April 2013.

Shannon C. E. Mathematical theory of communication. Bell Systems Technical Journal, No.127,(1948),pp.379-423.

SMLC. Implementing 21 st century smart manufacturing report.<https://smart-process-manufacturing.ucla.edu/>. 2013-03-02.

Tao F.,Zhang L.,Guo H., et al. Typical characteristics of cloud manufacturing and several key issues of cloud service composition. Computer Integrated Manufacturing Systems.Vol.17 No.3,(2011),pp.477-486.

Valilai O. F., Houshmand M.A. Collaborative and Integrated Platform to Support Distributed Manufacturing System Using a Service-oriented Approach Based on Cloud Computing Paradigm. Robotics and Computer Integrated Manufacturing.Vol.29,No.1 (2013),pp.110-128.

Wang H.Ch. DIT and Information. (2006)Beijing: Science Press. (in Chinese)

Wang H.Ch. Systems information measurement. University of Shanghai for Science and Technology.Vol.33,No.6 (2012),pp.631-640. (in Chinese)

Xiong Y.L., and Yin Zh.P. Digital manufacturing-the development direction of the manufacturing technology in the 21st century. Frontiers of Mechanical Engineering in China.Vol.1No.2(2006),pp.125-130.

Yang M., Qin Sh,J., and Chen Y. Research and application of integration mechanism based on resource. Computer Engineering and Technology (ICCET), 2nd International Conference on, IEEE, Vol.4(2010).pp.598-601

Yao X.F.,Yu M.,Chen Y.,et al. Connotation, architecture and key technology of Internet of manufacturing things. Computer Integrated Manufacturing Systems.Vol.20,No.1(2014),pp.1-10.

Zeng Y.,Wang J.,Wu X.X.et al. Research and Practice of Industrial Cloud Computing Platform. China Mechanical Engineering.Vol.23,No.1(2012),pp.69-74. (in Chinese)Zhang B.P. Manufacturing Informatics.(2003).Beijing: Tsinghua University Press. (in Chinese)

Zhang, L.,Guo, H.,Tao, F., et al.. Flexible management of resource service composition in cloud manufacturing. IEEE International Conference on Industrial Engineering and Engineering Management, Macao, (2010),pp.2278-2282.

Zhang L.,LUO Y.L.,FAN W.H., et al. Analyses of cloud manufacturing and related advanced manufacturing models. Computer Integrated Manufacturing Systems.Vol.17,No.3(2011),pp.458-468.

Zhou J., Digitalization and intelligentization of manufacturing industry. Adv. Manuf. Vol.1,No.1(2013),pp.:1-7. 